\begin{tabular}{|c|c|}
\hline Title & $\begin{array}{l}\text { Influence of pulse frequency on synthesis of nano and submicrometer spherical particles by pulsed laser melting in } \\
\text { liquid }\end{array}$ \\
\hline Author(s) & Sakaki, Shota; Ikenoue, Hiroshi; T suji, Takeshi; Ishikawa, Y oshie; Koshizaki, Naoto \\
\hline Citation & $\begin{array}{l}\text { A pplied surface science, } 435,529-534 \\
\text { https://doi.org/10.1016/. apsusc.2017.10.235 }\end{array}$ \\
\hline Issue Date & 2018-03-30 \\
\hline Doc URL & http:/hdl.handle.net/2115/77113 \\
\hline Rights & $\begin{array}{l}\text { (2) 2018. Ins manuscript versson is made avallable under the CC-BY-IVC-IVD } 4.0 \text { IIcense } \\
\text { http://reativecommons.org/icenses/by-nc-n/4.0/ }\end{array}$ \\
\hline Rights(URL) & http://creativecommons.org/icenses/by-nc-nd/4.0/ \\
\hline Type & article (author version) \\
\hline File Information & Manuscript_A pplSurfSci_revise.pdf \\
\hline
\end{tabular}

Instructions for use 


\title{
Influence of Pulse Frequency on Synthesis of Nano and Submicrometer Spherical Particles by Pulsed Laser Melting in Liquid
}

\author{
Shota Sakaki $^{\mathrm{a}, *}$, Hiroshi Ikenoue ${ }^{\mathrm{b}}$, Takeshi Tsuji ${ }^{\mathrm{c}}$, Yoshie Ishikawa ${ }^{\mathrm{d}}$, and Naoto Koshizaki ${ }^{\mathrm{a}, *}$ \\ ${ }^{a}$ Graduate School of Engineering, Hokkaido University, Kita 13, Nishi 8, Kita-ku, Sapporo, Hokkaido, 060-8628, \\ Japan \\ ${ }^{\mathrm{b}}$ Department of Gigaphoton Next GLP, Kyushu University, 744 Motooka, Nishi-ku, Fukuoka, 819-0395, Japan \\ ${ }^{c}$ Interdisciplinary Graduate School of Science and Engineering, Shimane University, 1060 Nishikawatsu-cho, \\ Matsue, Shimane, 690-8504, Japan \\ ${ }^{\mathrm{d}}$ Nanomaterials Research Institute, National Institute of Advanced Industrial Science and Technology (AIST), \\ Tsukuba Central 5, 1-1-1 Higashi, Tsukuba, Ibaraki, 305-8565, Japan
}

E-mail address: sakaki.shota@frontier.hokudai.ac.jp (S. Sakaki), koshizaki.naoto@eng.hokudai.ac.jp

$(\mathrm{N}$. Koshizaki)

\begin{abstract}
Submicrometer spherical particles (SMSPs) are reported to be fabricated by pulsed laser irradiation with a frequency of 10 or $30 \mathrm{~Hz}$ onto raw nanoparticles dispersed in liquid. Here, the effect of the pulse frequency on particles obtained by laser irradiation onto the suspension in a vessel, especially at higher pulse frequencies up to $800 \mathrm{~Hz}$, is investigated. At $200 \mathrm{~Hz}$ or lower, SMSPs of similar size can be fabricated, as at $10 \mathrm{or} 30 \mathrm{~Hz}$, by the same number of pulses. This indicates that the time required for particle fabrication can be greatly reduced and production efficiency can be improved using a high-frequency laser. In contrast, at $400 \mathrm{~Hz}$ or above, nanospherical particles (NSPs) are formed in addition to SMSPs, and the mass fraction of SMSPs is drastically decreased. This result suggests that consecutive laser pulse irradiation induces heat accumulation in particles and suspensions, resulting in a temperature increase and partial evaporation of the particles at $400 \mathrm{~Hz}$ or above. From the temperature increase of the suspension, the local temperature of the liquid surrounding the particles is believed to be increased by heat dissipation from the heated particles. Calculations suggest that an increase in the local liquid temperature would cause further heating of the particles.
\end{abstract}

\section{Keywords}

Zinc oxide; Pulsed laser melting; Colloids; Nanoparticles; Submicrometer particles

\section{Introduction}

In recent years, much attention has been focused on nanoparticle fabrication by pulsed laser ablation in liquid (PLAL) [1-6]. In PLAL, a bulk target immersed in liquid is irradiated with a high power pulsed laser, and nanoparticles are formed through explosive interaction between the bulk target and the laser pulses [5]. Size reduction of nanoparticles caused by pulsed laser fragmentation in liquid (PLFL) has also been investigated [6-12]. In PLFL, dispersed particles interact explosively with a high power pulsed laser, and nanoparticles are formed [12]. These techniques require fewer chemical reagents and no vacuum equipment.

SMSPs of various materials are fabricated by pulsed laser irradiation with relatively low laser fluence onto colloidal nanoparticles dispersed in liquid [6, 13-20]. In this method, a suspension in a glass vessel is generally irradiated for an appropriate time with magnetic stirring. Parts of the raw nanoparticles dispersed in the suspension are irradiated and absorb laser energy; SMSPs are then formed by particle melting, fusing, and quenching [20]. By repeating this process, almost all the agglomerates consisting of raw nanoparticles in the suspension are modified to form SMSPs. NSPs are also fabricated by this method at higher laser fluence than that used to form submicrometer particles [21]. Raw particles that absorb the laser energy are heated above the boiling point of the material and evaporated, resulting in NSP formation at relatively high laser fluence.

In this method, an Nd:YAG laser with a pulse frequency of several tens of hertz is usually used [15]. Therefore, laser irradiation at higher pulse frequencies is probably effective in reducing the total irradiation time required to treat almost all the raw particles in the vessel. In PLAL, ablated mass linearly increases with 
pulse frequency in the $\mathrm{kHz}$ and $\mathrm{MHz}$ regime until the temporal bubble-shielding effect arises [22, 23]. In PLML, kHz laser is also used to fabricate SMSPs [24]. However, the pulse frequency might affect particle cooling, because the interval between two consecutive laser pulses, that is, the maximum available time to cool particles heated over the melting point, would be comparable to the cooling time reported as $10^{-4}$ to $10^{-3} \mathrm{~s}$ when a high-frequency pulsed laser is used [25]. If the pulse interval is shorter than the cooling time, heat energy acquired from a single laser pulse cannot be completely dissipated within the pulse interval and will persist until a subsequent pulse arrives, resulting in a gradual temperature increase by heat accumulation in a particle even during the pulse interval. In this study, SMSPs are fabricated by KrF excimer laser irradiation at higher pulse frequencies than before to study the pulse frequency effect on the obtained particles.

\section{Experimental section}

Commercial ZnO particles (Sigma-Aldrich Co., LLC, $<100 \mathrm{~nm}$ ) were dispersed in deionized water at a concentration of $200 \mathrm{ppm}$. Then, $6 \mathrm{ml}$ of the suspension in a glass vessel was irradiated with a $\mathrm{KrF}$ excimer laser (Gigaphoton Inc., wavelength $248 \mathrm{~nm}$, pulse width $50 \mathrm{~ns}$ ) at various pulse frequencies (25-800 Hz). The beam size was $0.13 \mathrm{~cm}^{2}(1.5 \mathrm{~mm} \times 8.7 \mathrm{~mm}$ rectangle). The total number of pulses was fixed at 48,000 irrespective of the pulse frequency. Therefore, laser irradiation with a higher pulse frequency requires a shorter time to produce 48,000 pulses. The suspension temperature was measured with a thermocouple immersed in the liquid. After laser irradiation, the suspension was dropped onto a Si substrate to observe the particle morphology using a field-emission scanning electron microscope (FE-SEM, JSM-7001FA, JEOL Ltd.). To measure the size of the obtained SMSPs, the suspension was settled for a day to separate the NSPs from the produced SMSPs. A precipitate of the suspension was also dropped onto a Si substrate for FE-SEM observation.

\section{Results and discussion}

\subsection{Particle morphology}

SEM images of raw ZnO particles and particles obtained by $\mathrm{KrF}$ excimer laser irradiation at $100 \mathrm{~Hz}$ are presented in Fig. 1. Raw particles have angular morphology, and the average diameter of the raw particles is about $60 \mathrm{~nm}$ (Figure 1a). At a laser fluence of $182 \mathrm{~mJ}$ pulse $\mathrm{cm}^{-1}$, agglomerates of raw particles melted, and SMSPs with an average diameter of $324 \mathrm{~nm}$ were formed (Fig. 1(b)). In contrast, at a laser fluence of $203 \mathrm{~mJ} \mathrm{pulse}^{-1} \mathrm{~cm}^{-2}$, NSPs with an average diameter of $44 \mathrm{~nm}$ were formed in addition to SMSPs with an average diameter of $327 \mathrm{~nm}$ (Fig. 1(c)). In this case, agglomerates of raw $\mathrm{ZnO}$ particles would be vaporized by high-fluence laser irradiation. The vapor condenses and then solidifies to form spherical nanoparticles.

Fig. 2 shows SEM images of particles obtained after $\mathrm{KrF}$ excimer laser irradiation $\left(182 \mathrm{~mJ}\right.$ pulse $\left.^{-1} \mathrm{~cm}^{-2}\right)$ at various pulse frequencies. The time taken to produce 48,000 pulses is 1,920 seconds at $25 \mathrm{~Hz}, 240$ seconds at 200 $\mathrm{Hz}, 120$ seconds at $400 \mathrm{~Hz}$, and 60 seconds at $800 \mathrm{~Hz}$. At pulse frequencies of $25 \mathrm{~Hz}$ to $200 \mathrm{~Hz}$, almost all the obtained particles were SMSPs (Figs. 2(a) and 2(b)). In contrast, at a pulse frequency of $400 \mathrm{~Hz}$ and above (Figs.

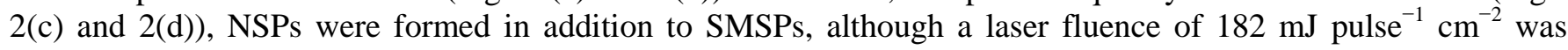
insufficient to obtain NSPs by vaporization at a pulse frequency of $100 \mathrm{~Hz}$, as indicated in Fig. 1. This result suggests that heat energy absorbed by a particle is not completely dissipated during the pulse interval and subsequently accumulates with consecutive laser pulses. Figs. 3(a-d) shows SEM images of ZnO particle precipitates by natural sedimentation of the suspension irradiated with a $\mathrm{KrF}$ excimer laser at various pulse frequencies as depicted in Fig. 2. NSPs were easily separated from the suspension by natural sedimentation over one day. By counting the SMSPs in the SEM images of Figs. 3(a-d), histograms of particle diameter distribution are graphed, respectively (Figs. 3(e-f)).

Fig. 4(a) shows the pulse frequency dependence of the mass fraction of SMSPs in the products estimated from SEM images shown in Fig. 2. The mass fraction of the SMSPs to the all particles (SMSPs and NSPs) in the products was estimated by converting the size distribution data of all the SMSPs and NSPs in the SEM images into 
mass ratio of SMSPs to NSPs assuming that all particles are spherical and no compositional change from ZnO. Since the particle mass is proportional to the cube of the particle diameter, SMSPs are much heavier than NSPs. Before natural sedimentation, most particles obtained were SMSPs at a pulse frequency of 25 to $200 \mathrm{~Hz}$. In contrast, at $400 \mathrm{~Hz}$ or higher, the mass fraction of SMSPs was drastically decreased through vaporization. Therefore, laser irradiation at a pulse frequency of $200 \mathrm{~Hz}$ and below is appropriate for effectively obtaining SMSPs. By natural sedimentation over one day, we could remove NSPs from the suspension and prepare an SMSP suspension with a mass fraction of over $90 \%$.

The pulse frequency dependence on the average diameter of SMSPs measured from the histograms of particle diameter distribution (Figs. 3(e-h)) at a laser fluence of $182 \mathrm{~mJ}$ pulse $\mathrm{cm}^{-2}$ is depicted in Fig. 4(b). For a pulse frequency range where mainly SMSPs are obtained, as shown in Fig. $2(\leq 200 \mathrm{~Hz})$, obtained particles have similar sizes of about $300 \mathrm{~nm}$. Thus, a larger pulse frequency up to $200 \mathrm{~Hz}$ is effective in reducing the irradiation time for SMSP synthesis. Above the threshold for NSP formation in Fig. 2 ( $\geq 400 \mathrm{~Hz})$, however, the obtained large particles have a submicrometer size of about $240 \mathrm{~nm}$, which is smaller than those obtained at a lower pulse frequency $(\leq 200$ $\mathrm{Hz}$ ). This result suggests that particles irradiated with a pulsed laser at high frequency might be heated, resulting in size reduction of the SMSPs due to partial evaporation.

Fig. 4(b) also shows the pulse frequency dependence on the average diameter of NSPs measured from SEM images in Fig. 2. The average particle diameter decreases with the pulse frequency above the threshold for NSP formation determined from Fig. $2(\geq 400 \mathrm{~Hz}$ ). The average diameter of nanoparticles obtained by pulsed laser irradiation is reported to gradually decrease as the laser fluence increases $[9,10]$. Therefore, above the threshold for NSP formation, the effect of the pulse frequency on the change in nanoparticle size would correspond to the effect of laser fluence reported earlier. Heat accumulation resulting from consecutive high-frequency laser pulses would cause further heating of the particles.

\subsection{Liquid temperature}

The average suspension temperature during laser irradiation is gradually elevated by heat dissipation from the particles that absorb laser energy to the surrounding liquid. Fig. 5 shows the suspension temperature increase in a glass vessel during laser irradiation at various pulse frequencies. The highest attained suspension temperature increases with pulse frequency because the input energy per unit time increases in proportion to the pulse frequency. However, the temperature of the liquid adjacent to the particles in the laser-irradiated region is higher than that of the laser-irradiated region remote from the particles, because the particles irradiated with laser pulses are the heat source for the suspension. Therefore, the temperature of the liquid adjacent to the particles is much higher than the average suspension temperature, and adjacent liquid would probably evaporate depending on the laser irradiation conditions, especially at the high input energy per unit time accompanying a high pulse frequency. Such a high liquid temperature would induce vaporization of the liquid at the interface and affect the highest attained temperature discussed in the following section.

\subsection{Calculation of particle temperature}

These experimental results suggest that the heat energy absorbed from a single laser pulse is not completely dissipated before the arrival of the subsequent laser pulse, and it accumulates in a particle as a result of consecutive laser pulses at high frequencies. Thus, the time required to completely dissipate the energy supplied by a laser pulse is estimated to be longer than $2.5 \mathrm{~ms}$, which is the corresponding laser pulse interval for a pulse frequency of 400 Hz. However, this cooling time is much longer than the value estimated by conductive heat transfer calculation using the particle heating-cooling model [26]. The heat energy dissipated from the particles $q_{p}$ is described by the following equation:

$\frac{d q_{p}}{d t}=h \cdot \pi d^{2} \cdot\left\{T_{p}-T_{0}\right\}$

where

$h=\frac{N u_{d} \cdot k}{d}$

Here, $h$ is the heat transfer coefficient, $d$ is the particle diameter, $T_{p}$ is the particle temperature, $T_{0}$ is the 
temperature of the surrounding liquid, $N u_{d}$ is the Nusselt number, and $k$ is the heat conductivity of the surrounding liquid or vapor generated by liquid evaporation. On the submicrometer scale, the Nusselt number is constant at $N u_{d} \approx 2$, because the convection around a submicrometer particle is negligible [27, 28]. When the particles are cooled by evaporated liquid, the heat transfer coefficient of particles $300 \mathrm{~nm}$ in diameter can be estimated as $0.29 \mathrm{MW} \mathrm{m}^{-2} \mathrm{~K}^{-1}$ from Eq. (2). In this case, particles heated to the melting point are cooled to the temperature of the surrounding liquid on a microsecond time scale. Therefore, a pulse interval of $2.5 \mathrm{~ms}$ is sufficient for particle cooling.

The heat energy accumulated in the particles $E_{p}$ is the difference between particle heating by laser absorption and particle cooling by conductive heat transfer.

$\frac{d E_{p}}{d t}=Q_{a b s}^{\lambda} \cdot \frac{\pi d^{2}}{4} \cdot J(t)-\frac{d q_{p}}{d t}$

Here, $Q_{a b s}^{\lambda}$ is the absorption efficiency, $\frac{\pi d^{2}}{4}$ is the particle geometric cross section, and $J(t)$ is the time-dependent laser fluence. The absorption efficiency of SMSPs is calculated from refractive index and extinction coefficient using Mie theory [25, 29].

The particle temperature $T_{p}$ below the melting point, at the melting point, and above the melting point is defined by the following equations.

$T_{p}(t)=T_{0}+\frac{1}{\rho_{p} \cdot \frac{\pi d^{3}}{6} \cdot c_{s}} \cdot E(t)$

$T_{p}(t)=T_{m}$

$T_{p}(t)=T_{\mathrm{m}}+\frac{1}{\rho_{p} \frac{\pi d^{3}}{6} \cdot c_{l}} \cdot\left[E(t)-\rho_{p} \cdot \frac{\pi d^{3}}{6} \cdot\left(H_{T_{m}}-H_{T_{0}}+\Delta H_{m}\right)\right]$

Here, $\rho_{p}$ is the density of the particle, $\frac{\pi d^{3}}{6}$ is the particle volume, $c_{S}$ is the particle specific heat in the solid state, $T_{m}$ is the melting point of the material, $H_{T_{m}}-H_{T_{0}}$ is the relative enthalpy required for the start of melting, $\Delta H_{m}$ is the latent enthalpy of melting, and $c_{l}$ is the particle specific heat in the liquid state.

Fig. 6 shows the calculated highest attained temperature of a $\mathrm{ZnO}$ spherical particle $250 \mathrm{~nm}$ in diameter irradiated with a nanosecond laser (pulse width $50 \mathrm{~ns}$, laser fluence $182 \mathrm{~mJ} \mathrm{pulse}^{-1} \mathrm{~cm}^{-2}$ ). The highest attained temperature is elevated by an increase in the temperature of the surrounding liquid because this increases the initial particle temperature. Moreover, the highest attained temperature of the particles constantly cooled by a liquid vapor film (red line) was higher than that of the particles cooled by a liquid undergoing a phase transition from liquid to vapor (black line). When using a laser with a pulse width of several tens of nanoseconds, the particles dissipate heat energy to the surrounding liquid because heated particles are cooled by a liquid with high heat conductivity during the initial stage of heating [26]. Therefore, a low-heat-conductive vapor film around the particles generated by heat accumulation reduces heat dissipation.

Calculations suggest that the highest attained temperature of a particle increases by a few hundred $\mathrm{K}$ through heat accumulation in the adjacent liquid. The actual attained temperature of particles irradiated with a pulsed laser at a laser fluence of $182 \mathrm{~mJ}$ pulse ${ }^{-1} \mathrm{~cm}^{-2}$ would be near the boiling point because NSPs were formed at a laser fluence of $203 \mathrm{~mJ}$ pulse $\mathrm{cm}^{-2}$ (Fig. 1). Therefore, heat accumulation in the liquid adjacent to particles may cause heating of the particles to the boiling point, resulting in nanoparticle formation at a pulse frequency of above 400 $\mathrm{Hz}$.

\section{Conclusions}

SMSPs were fabricated by irradiation with a KrF excimer laser (wavelength $248 \mathrm{~nm}$, pulse width $50 \mathrm{ns)}$ onto 6 $\mathrm{ml}$ of the suspension with a particle concentration of $200 \mathrm{ppm}$ in a glass vessel. The total time of laser irradiation for the synthesis of SMSPs can be reduced using a high-frequency pulsed laser at a laser fluence of $182 \mathrm{~mJ} \mathrm{pulse}^{-1}$ $\mathrm{cm}^{-2}(\leq 200 \mathrm{~Hz})$. The size of the obtained SMSPs is similar in this frequency range. In contrast, NSPs and SMSPs are simultaneously formed using a pulsed laser with a frequency of $400 \mathrm{~Hz}$ or higher. This result suggests that the 
laser energy is accumulated in particles and liquid by consecutive laser pulses, resulting in partial evaporation of the particles above the pulse frequency threshold $(\geq 400 \mathrm{~Hz})$. Through this vaporization process, the average diameter of the obtained SMSPs decreased. However, the time required to cool particles from the melting point to ambient temperature is much shorter than $2.5 \mathrm{~ms}$ (pulse interval for a pulse frequency of $400 \mathrm{~Hz}$ ). Therefore, nanoparticle formation was attributed to an increase in the suspension temperature. The average suspension temperature was elevated during laser irradiation, especially at a high pulse frequency. The liquid temperature adjacent to the particles is higher than the average suspension temperature because the particles irradiated with laser pulses are the heat source for the suspension. Calculations suggest that the highest attained temperature of the particles increases by a few hundred $\mathrm{K}$ through heat accumulation in the adjacent liquid, resulting in nanoparticle formation at a high pulse frequency.

\section{Acknowledgment}

This work was supported by JSPS KAKENHI Grant Numbers 26289266 and 26870908.

\section{References}

[1] P.P. Patil, D.M. Phase, S.A. Kulkarni, S.V. Ghaisas, S.K. Kulkarni, S.M. Kanetkar, S.B. Ogale, V.G. Bhide, Phys. Rev. Lett. 58 (1987) 238-241.

[2] J. Neddersen, G. Chumanov, T.M. Cotton, Appl. Spectrosc. 47 (1993) 1959-1964.

[3] B.G. Ershov, E. Janata, A. Henglein, J. Phys. Chem. 97 (1993) 339-343.

[4] F. Mafuné, J. Kohno, Y. Takeda, T. Kondow, J. Phys. Chem. B 104 (2000) 9111-9117.

[5] C. Liang, Y. Shimizu, M. Masuda, T. Sasaki, N. Koshizaki, Chem. Mater. 16 (2004) 963-965.

[6] D. Zhang, B. Gökce, S. Barcikowski, Chem. Rev. 117 (2017) 3990-4103.

[7]P.V. Kamat, J. Phys. Chem. B 106 (2002) 7729-7744.

[8] D. Werner, A. Furube, T. Okamoto, S. Hashimoto, J. Phys. Chem. C 115 (2011) 8503-8512.

[9] D. Werner, T. Ueki, S. Hashimoto, J. Phys. Chem. C 116 (2012) 5482-5491.

[10] D. Werner, S. Hashimoto, Langmuir 29 (2013) 1295-1302.

[11] P. Boyer, D. Ménard, M. Meunier, J. Phys. Chem. C 114 (2010) 13497-13500.

[12] H. Usui, T. Sasaki, N. Koshizaki, J. Phys. Chem. B 110 (2006) 12890-12895.

[13] Y. Ishikawa, Y. Shimizu, T. Sasaki, N. Koshizaki, Appl. Phys. Lett. 91 (2007) 161110.

[14] H. Wang, M. Miyauchi, Y. Ishikawa, A. Pyatenko, N. Koshizaki, Y. Li, L. Li, X. Li, Y. Bando, D. Golberg, J. Am. Chem. Soc. 133 (2011) 19102-19109.

[15] H. Wang, N. Koshizaki, L. Li, L. Jia, K. Kawaguchi, X. Li, A. Pyatenko, Z. Swiatkowska-Warkocka, Y. Bando, D. Golberg, Adv. Mater. 23 (2011) 1865-1870.

[16] T. Tsuji, T. Yahata, M. Yasutomo, K. Igawa, M. Tsuji, Y. Ishikawa, N. Koshizaki, Phys. Chem. Chem. Phys. 15 (2013) 3099-3107.

[17] X. Li, N. Koshizaki, A. Pyatenko, Y. Shimizu, H. Wang, J. Liu, X. Wang, M. Gao, Z. Wang, X. Zeng, Opt. Express 19 (2011) 2846-2851.

[18] X. Li, A. Pyatenko, Y. Shimizu, H. Wang, K. Koga, N. Koshizaki, Langmuir 27 (2011) 5076-5080.

[19] K. Kawasoe, Y. Ishikawa, N. Koshizaki, T. Yano, O. Odawara, H. wada, Appl. Phys. B 119 (2015) 475-483.

[20] H. Wang, A. Pyatenko, K. Kawaguchi, X. Li, Z. Swiatkowska-Warkocka, N. Koshizaki, Angew. Chem. Int. Ed. 49 (2010) 6361-6364.

[21] Y. Ishikawa, N. Koshizaki, A. Pyatenko, N. Saitoh, N. Yoshizawa, Y. Shimizu, J. Phys. Chem. C 120 (2016) 2439-2446.

[22] C. L. Sajti, R. Sattari, B. N. Chichkov, S. Barcikowski, J. Phys. Chem. C 114 (2010) 2421-2427.

[23] R. Streubel, S. Barcikowski, B. Gökce, Opt. Lett. 41 (2016) 1486-1489.

[24] D. Zhang, M. Lau, S. Lu, S. Barcikowski, B. Gökce, Sci. Rep. 7 (2017) 40355.

[25] A. Pyatenko, H. Wang, N. Koshizaki, T. Tsuji, Laser Photonics Rev. 7 (2013) 596-604.

[26] S. Sakaki, H. Ikenoue, T. Tsuji, Y. Ishikawa, N. Koshizaki, Chem. Phys. Chem. 18 (2017) 1101-1107.

[27] A.F. Mills, Heat Transfer, 2nd ed., Prentice Hall, New York, 1998, pp. 275-282.

[28] J.S. Donner, G. Baffou, D. McCloskey, R. Quidant, ACS Nano 5 (2011) 5457-5462.

[29] G. Mie, Ann. Phys. 330 (1908) 377-445. 


\section{Figure Captions}

Fig. 1. SEM images of (a) raw $\mathrm{ZnO}$ particles and particles irradiated with a $\mathrm{KrF}$ excimer laser $(100 \mathrm{~Hz}$ ) at a laser fluence of (b) $182 \mathrm{~mJ}$ pulse $\mathrm{cm}^{-2}$ and (c) $203 \mathrm{~mJ}_{\text {pulse }} \mathrm{cm}^{-2}$.

Fig. 2. SEM images of $\mathrm{ZnO}$ particles irradiated with a $\mathrm{KrF}$ excimer laser at a laser fluence of $182 \mathrm{~mJ} \mathrm{pulse}^{-1} \mathrm{~cm}^{-2}$. Pulse frequency of (a) $25 \mathrm{~Hz}$, (b) $200 \mathrm{~Hz}$, (c) $400 \mathrm{~Hz}$, and (d) $800 \mathrm{~Hz}$.

Fig. 3. SEM images of $\mathrm{ZnO}$ particle precipitates by natural sedimentation of the suspension irradiated with a $\mathrm{KrF}$ excimer laser irradiation at a laser fluence of $182 \mathrm{~mJ}_{\text {pulse }} \mathrm{cm}^{-2}$. Pulse frequency of (a) $25 \mathrm{~Hz}$, (b) $200 \mathrm{~Hz}$, (c) $400 \mathrm{~Hz}$, and (d) $800 \mathrm{~Hz}$. The corresponding particle diameter distributions of (a-d) are shown in (e-f), respectively.

Fig. 4. Pulse frequency dependence on (a) mass fraction of SMSPs in the products and (b) average particle diameter measured by counting only ZnO SMSPs in Fig. 3 and only NSPs in Fig. 2 obtained by nanosecond pulsed KrF excimer laser irradiation at a laser fluence of $182 \mathrm{~mJ} \mathrm{pulse}^{-1} \mathrm{~cm}^{-2}$.

Fig. 5. Increase in the suspension temperature during laser irradiation at various pulse frequencies measured by thermocouple immersed in the liquid.

Fig. 6. Highest attained temperature of SMSPs $250 \mathrm{~nm}$ in diameter irradiated with a nanosecond laser with pulse width of 50 ns at a laser fluence of $182 \mathrm{~mJ}$ pulse $\mathrm{cm}^{-1}$. Cooling by surrounding liquid undergoing phase transition from liquid to vapor (Black line), and cooling by surrounding vapor generated by liquid evaporation (Red line).

\section{Highlights}

Submicrometer spherical particles were fabricated at various pulse frequencies.

Production efficiency was improved by a high-frequency laser.

Spherical nanoparticles were formed above the pulse frequency threshold.

. The effect of the liquid temperature on the pulsed laser heating was estimated. 